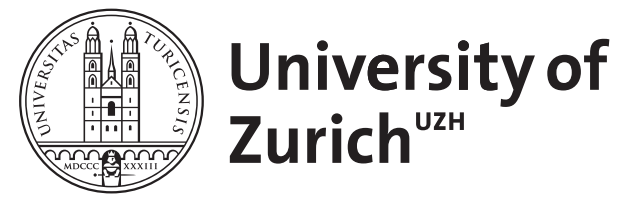

\title{
Stand und Perspektiven der Erforschung des päpstlichen Legatenwesens im
} Hochmittelalter

\author{
Zey, Claudia
}

Posted at the Zurich Open Repository and Archive, University of Zurich ZORA URL: https://doi.org/10.5167/uzh-70446

Book Section

Originally published at:

Zey, Claudia (2012). Stand und Perspektiven der Erforschung des päpstlichen Legatenwesens im Hochmittelalter. In: Johrendt, Jochen; Müller, Harald. Rom und die Regionen : Studien zur Homogenisierung der lateinischen Kirche im Hochmittelalter. Göttingen: De Gruyter, 157-166. 


\title{
Stand und Perspektiven der Erforschung des päpstlichen Legatenwesens im Hochmittelalter
}

\author{
Claudia ZeY
}

Die Erforschung des päpstlichen Legatenwesens ist seit einigen Jahren wieder en vogue. Die Tagungsfrequenz mit Themen zu päpstlicher Diplomatie, zur kirchlichen Kommunikation und zum Kardinalat im Allgemeinen ${ }^{1}$ sowie zum Legationswesen im Besonderen ${ }^{2}$ hat in den letzten Jahren deutlich zugenommen und scheint einstweilen auch nicht abzureißen ${ }^{3}$. Die zeitliche Ausrichtung

1 Tagungen zum Thema Diplomatie, Gesandtschaftswesen und Kardinalat (Beispiele): „Aus der Frühzeit europäischer Diplomatie. Zum geistlichen und weltlichen Gesandtschaftswesen vom 12. bis zum 15. Jahrhundert", veranstaltet von Claudia Märtl und Claudia Zey in Zürich vom 26.-28.9.2007. Der Tagungband mit identischem Titel wurde 2008 publiziert. - „Die Kardinäle des Mittelalters und der frühen Renaissance. Integration, Kommunikation, Habitus“, veranstaltet von Jürgen Dendorfer und Ralf Lützelschwab in Rom vom 1.-2.7.2009. Die Publikation des Tagungsbandes wird vorbereitet. Der Band „Zentrum und Netzwerk. Kirchliche Kommunikation und Raumstrukturen im Mittelalter“, hg. v. Gisela DrossBACH/Hans-Joachim SCHMIDT, Berlin/New York 2008 (Scrinium Fribourgense 22) geht auf eine Sektion beim Kieler Historikertag 2004 zurück. Und auch der im Rahmen dieses Netzwerks entstandene Sammelband „Römisches Zentrum und kirchliche Peripherie. Das universale Papsttum als Bezugspunkt der Kirchen von den Reformpäpsten bis zu Innozenz III.“, hg. v. Jochen Johrendt/Harald MÜller, Berlin/New York 2008 (Neue AAG 2) hatte eine Tagung in Rom 2006 als Vorläufer.

2 Tagungen zum päpstlichen Legatenwesen oder bei denen auch das päpstliche Legatenwesen zur Sprache kam (Beispiele): „Les légats pontificaux. Paix et unité de l'Eglise, de la restructuration grégorienne à l'aube du Concile de Trente $\left(\mathrm{mi} \mathrm{XI}{ }^{\mathrm{e}}-\mathrm{mi} \mathrm{XVI}\right.$ siècle)“, veranstaltet von Hélène Millet und Pascal Montaubin in Paris vom 12.14.2.2009. Die Publikation des Tagungsbandes wird vorbereitet. „Schismes, dissidences, oppositions: la France et le Saint-Siège avant Boniface VIII", 6. Table ronde zur Gallia Pontificia veranstaltet von Bernard Barbiche und Rolf Grosse in Paris am 29.5.2009. Die Publikation des Tagungsbandes wird vorbereitet. „Legati e delegati: le carriere e gli ambiti di azione (secoli XII-XIII) / Päpstliche Legaten und Delegaten: Forschungsstand und Forschungsfragen (12.-13. Jahrhundert)" veranstaltet von Maria Pia Alberzoni und Claudia Zey in Mailand vom 5.-6.6.2009. Der Tagungsband wurde unter dem Titel "Legati e delegati papali nei secoli XII e XIII" 2012 publiziert. "Legationen und Reisen - Mittel der päpstlichen Integration im 11. und 12. Jahrhundert", veranstaltet von Klaus Herbers in Erlangen am 16.10.2009.

3 Folgende für 2010 geplante Tagungen sind mir bekannt: „Die Ordnung der Kommunikation und die Kommunikation der Ordnungen im mittelalterlichen Europa. Zent- 
dieser Veranstaltungen gilt Hoch- und Spätmittelalter gleichermaßen, obwohl der Stand der Erforschung des päpstlichen Legatenwesens in beiden Epochenabschnitten unterschiedlicher nicht sein könnte. Für das Spätmittelalter steckt die Erforschung des Legatenwesens noch in den Anfängen. Geographische oder chronologische Überblicksdarstellungen fehlen fast gänzlich und sind auch angesichts des überbordenden und noch kaum ausgewerteten Quellenmaterials in absehbarer Zeit nicht zu leisten ${ }^{4}$.

\section{Stand der Erforschung des hochmittelalterlichen Legatenwesens}

Das hochmittelalterliche Legatenwesen ist bereits seit der Wende vom 19. zum 20. Jahrhundert Gegenstand rechtsgeschichtlicher und historischer Forschungen mit einem frühen Kulminationspunkt in der ersten Hälfte des 20. Jahrhunderts durch zahlreiche, hauptsächlich von Albert Brackmann, dem maßgeblichen Bearbeiter der Germania Pontificia, und Wilhelm Levison angeregte Dissertationen ${ }^{5}$. Weg von der normativen Erforschung (wenn auch nicht im

ralität: Papsttum und Orden im Europa des 12. und 13. Jahrhunderts“, veranstaltet von Cristina Andenna, Klaus Herbers und Gert Melville in der Villa Vigoni vom 16.19.6.2010. „El papado limitado. Legados - jueces - limites“, veranstaltet von Klaus Herbers und Fernando López Alsina in Lissabon vom 8.-11.7.2010. „Aux origines d'une diplomatie méditerranéenne. Les ambassadeurs, moyens humains de la diplomatie (Antiquité romaine et Haut Moyen-Âge), wird veranstaltet von Audrey BeckerPiriou und Nicolas Drocourt in Metz vom 14.-16.10.2010.

4 Zum Stand und zu den Perspektiven der Erforschung des spätmittelalterlichen Legatenwesens vgl. Werner MALECZEK: Die päpstlichen Legaten im 14. und 15. Jahrhundert, in: Gesandtschafts- und Botenwesen im spätmittelalterlichen Europa, hg. v. Rainer C. SCHWINGES/Klaus WRIEDT, Ostfildern 2003 (VuF 60), S. 33-86. Den Forschungsstand zum Legatenrecht im Spätmittelalter thematisiert auch Birgit STUDT: Legationen als Instrumente päpstlicher Reform- und Kreuzzugspropaganda im 15. Jahrhundert, in: Formen und Funktionen öffentlicher Kommunikation im Mittelalter, hg. v. Gerd ALthofF, Stuttgart 2001 (VuF 51), S. 421-453.

5 Die Arbeiten werden im Folgenden nicht einzeln aufgelistet. Das ist bereits in vielen Übersichten zur Forschung geschehen, vgl. Hans OLLENDIEK: Die päpstlichen Legaten im deutschen Reichsgebiet von 1261 bis zum Ende des Interregnums, Freiburg i. Ü. 1976 (Historische Schriften der Universität Freiburg 3), S. 23-34; Werner MALECZEK: Papst und Kardinalskolleg von 1191 bis 1216. Die Kardinäle unter Coelestin III. und Innocenz III., Wien 1984 (Publikationen des Historischen Instituts beim Österreichischen Kulturinstitut in Rom 1/6), S. 336; Ma Tapio SAlminen: In the Pope's Clothes: Legatine Representation and Apostolical Insignia in High Medieval Europe, in: Roma, Magistra Mundi. Itineraria culturae medievalis - Parvi flores. Mélanges offerts au Père L. E. Boyle à l'occasion de son 75e anniversaire, hg. v. Jacqueline HAMESSE, Louvain-La-Neuve 1998 (Fédération Internationale des Instituts d'Études Médiévales. Textes et études du moyen âge 10, 3), S. 339-354, hier S. 341f.; Stefan WeISS: Die Urkunden der päpstlichen Legaten von Leo IX. bis Coelestin III. (10491198), Köln u.a. 1995 (Forschungen zur Kaiser- und Papstgeschichte des Mittelalters. 
Gegensatz zu ihr) galt die Aufmerksamkeit dieser Qualifikationsschriften vornehmlich der Legationspraxis im Zielland. Für die Zeitspanne bis 1198 wurden Arbeiten über das päpstliche Legatenwesen in Deutschland, Frankreich, England und auf der Iberischen Halbinsel verfasst. Partiell wurden daneben Oberund Mittelitalien sowie das süditalische Normannenreich berücksichtigt. In späteren Jahrzehnten kamen weitere einschlägige Arbeiten zu Skandinavien, Frankreich, England ${ }^{6}$, Schottland ${ }^{7}$ und Irland, der Iberischen Halbinsel, den Kreuzfahrerstaaten und Teilen Ostmitteleuropas ${ }^{8}$ hinzu. Diesen Arbeiten gebührt das große Verdienst, erstmals das urkundliche und historiographische Quellenmaterial kritisch gesichtet und aufgearbeitet zu haben. Deswegen bleiben sie (trotz unterschiedlicher Qualität im Einzelnen) Grundlage für die weitere Erforschung des Phänomens im Hochmittelalter. In dem Bemühen um eine möglichst umfassende Darstellung wurden mehrere Perspektiven miteinander verwoben: Wichtige politische Ereignisse dienten zur chronologischen Einbettung der Untersuchung, die Grobgliederung erfolgte nach Pontifikaten, die Binnengliederung nach Legaten, während die Darstellung zwischen personen- und regionalgeschichtlichem Blickwinkel schwankt. Eine systematische Auswertung des so bereitgestellten Stoffs wurde im Bewusstsein der Autorinnen und Autoren, einen Baustein zu einer noch zu schreibenden Gesamtdarstellung $\mathrm{zu}$ schaffen, nur ansatzweise unternommen. Alle Arbeiten bieten jedoch etwas zur rechtlichen Stellung der Legaten, ihrer begrifflichen Differenzierung, ihren Vollmachten, ihrer kirchenpolitischen und ,politischen“ Tätigkeit, zur Urkundenausstellung, zum Zeremoniell, zur Zusammensetzung der Legationen, zur Dauer, zum Verlauf und zum Gefolge.

Trotz dieser weit gediehenen Vorarbeiten ist bisher noch wenig Zusammenfassendes zum Legationswesen im Hochmittelalter insgesamt publiziert worden ${ }^{9}$. Vielmehr wurde weiterhin mit vielen neuen Erkenntnissen im Detail chronologisch $^{10}$ und geographisch ${ }^{11}$ eingeschränkt geforscht.

Beih. zu J. F. Böhmer, RI 13), S. 5-11; MALECzeK: Legaten (wie Anm. 4) S. 35 f.; zuletzt Achim Thomas HACK: Codex Carolinus. Päpstliche Epistolographie im 8. Jahrhundert, 2 Bde., Stuttgart 2006 (Päpste und Papsttum 35), Bd. 1 S. 489-497.

6 Die Dissertation von Ilicia J. SPREY: Papal Legates in English Politics, 1100-1272 (Diss.) Virginia 1998, scheint nicht publiziert worden zu sein.

7 Paul Craig Ferguson: Medieval Papal Representatives in Scotland: Legates, Nuncios, and Judges-Delegate, 1125-1286, Edinburgh 1997 (Publications of the Stair Society 45).

8 Vgl. Reinhard WENSKUs: Zu einigen päpstlichen Legationen nach Böhmen und Mähren im 12. Jahrhundert, in: ZKG 70 (1959) S. 141-146. Für Dalmatien, Kroatien und Ungarn ist der Band von Lothar WALDMÜLLER: Die Synoden in Dalmatien, Kroatien und Ungarn von der Völkerwanderung bis zum Ende der Arpaden (1311), Paderborn 1987 (Konziliengeschichte, Reihe A: Darstellungen 4), recht ergiebig. Vgl. zu Ungarn jetzt Gergely Kiss: Les aspects des activitiés des légats pontificaux en Hongrie aux $\mathrm{XI}^{\mathrm{e}}-$ XIII ${ }^{\mathrm{e}}$ siècles, in: Chronica (Szeged) 9 (2011), S. 37-53.

9 Vgl. Ian Stuart RoBinson: The Papacy, 1073-1198. Continuity and Innovation, Cambridge 1990 (Cambridge Medieval Textbooks), S. 146-178: Papal Legates. Bis da- 
to unpubliziert ist meine 2002 abgeschlossene Habilitationsschrift zur päpstlichen Legatenpolitik im 11. und 12. Jahrhundert. Wichtige Ergebnisse daraus wurden in zwei Aufsätzen publiziert: Claudia ZEY: Die Augen des Papstes. Zu Eigenschaften und Vollmachten päpstlicher Legaten, in: JOHRENDT/MÜLLER (wie Anm. 1) S. 77-108; DIES.: Handlungsspielräume - Handlungsinitiativen. Aspekte der päpstlichen Legatenpolitik im 12. Jahrhundert, in: DrossBACH/SCHMIDT (wie Anm. 1) S. 63-92.

Für das 13. Jahrhundert wurde von Ollendiek (wie Anm. 5) S. 36, zunächst die Erforschung auf regionaler Ebene angemahnt wie sie für das 12. Jahrhundert bereits existiert. Auf England beschränkt gibt es für das 13. Jahrhundert immerhin zwei Dissertationen: Ann UNDERHILL: Papal Legates to England in the Reign of Henry III (12161272), (Diss.) Bloomington 1965; Rose C. Clifford: England as a Papal Fief. The Role of the Papal Legate in the Early Period. 1216-1241, (Diss.) Los Angeles 1972. Gänzlich unergiebig für das Mittelalter sind die Abhandlungen zum päpstlichen Gesandtschaftswesen von den Anfängen bis in die Neuzeit: Igino CARDINALE: Le SaintSiège et la diplomatie. Aperçu historique, juridique et pratique de la diplomatie pontificale, Paris u.a. 1962; Mario Oliveri: Natura e funzioni dei legati pontifici nella storia e nel contesto ecclesiologico del Vaticano II, Città del Vaticano ${ }^{2} 1982$ (Storia e attualità 8); Pierre BLET: Histoire de la représentation diplomatique du Saint Siège des origines à l'aube du XIX ${ }^{\mathrm{e}}$ siècle, Città del Vaticano 1982 (21990) (Collectanea archivi Vaticani 9).

10 In folgenden Monographien zu einzelnen Päpsten ist deren Legatenpolitik eigens behandelt: H. E. J. CowDREY: Pope Gregory VII 1073-1085, Oxford 1998, S. 592596, mit einem knappen Kapitel zum Legatenwesen und ausfuhrlicheren Darlegungen im länderspezifischen Teil; Uta-Renate BlumenTHAL: Gregor VII. Papst zwischen Canossa und Kirchenreform, Darmstadt 2001 (Gestalten des Mittelalters und der Renaissance), S. 202-220; Alfons BECKER: Papst Urban II. (1088-1099), Teil 1: Herkunft und kirchliche Laufbahn. Der Papst und die lateinische Christenheit, Stuttgart 1964 (MGH Schriften 19/1); Teil 2: Der Papst, die griechische Christenheit und der Kreuzzug, Stuttgart 1988 (MGH Schriften 19/2), mit der Behandlung einzelner Legationen. Ein gesondertes Kapitel zum Legatenwesen soll im dritten Band erscheinen; Carlo Servatius: Paschalis II. (1099-1118). Studien zu seiner Person und seiner Politik, Stuttgart 1979 (Päpste und Papsttum 14), S. 42-69, zu Kurie und Kardinalskollegium und der Berücksichtigung der Legationen in der fortlaufenden Darstellung; Beate SCHILling: Guido von Vienne - Papst Calixt II., Hannover 1998 (MGH Schriften 45), S. 354-389, zur Berücksichtigung der Legationstätigkeit unter Calixt in der fortlaufenden Darstellung sowie einem Kapitel zum Kardinalskollegium dieses Papstes (S. 549-564); Mary STROLL: Calixtus II (1119-1124). A Pope born to rule (Studies in the history of Christian traditions 116), Leiden 2004, mit ausführlicher Erwähnung der Legationen in den länderspezifischen Kapiteln; Michael HORN: Studien zur Geschichte Papst Eugens III. (1145-1153), Frankfurt a. M. u.a. 1992 (Europäische Hochschulschriften 3/508), S. 208-220.

11 Für Frankreich: Rudolf HiestAND: Les légats pontificaux en France du milieu du XI ${ }^{\mathrm{e}}$ à la fin du XII ${ }^{\mathrm{e}}$ siècle, in: L'église de France et la papauté $\left(\mathrm{X}^{\mathrm{e}}-\mathrm{XIII}{ }^{\mathrm{e}}\right.$ siècle) / Die französische Kirche und das Papsttum (10.-13. Jahrhundert). Actes du XXVI ${ }^{\mathrm{e}}$ colloque historique franco-allemand organisé en coopération avec l'École Nationale des Chartes par l'Institut Historique Allemand de Paris (Paris, 17-19 octobre 1990), hg. v. Rolf Grosse, Bonn 1993 (Studien und Dokumente zur Gallia Pontificia 5), S. 54-80; Rolf GROSSE: La fille aînée de l'Église: Frankreichs Kirche und die Kurie im 12. Jahrhundert, in: Johrendt/Müller (wie Anm. 1) S. 299-321; Pascal Montaubin: De pe- 
Neben den breiter angelegten rechts- und allgemeingeschichtlichen Untersuchungen zum Legatenwesen entstanden eine Reihe von personengeschichtlichen Arbeiten, deren zeitlicher Schwerpunkt bedingt durch die bessere Quellenlage allerdings im 13. Jahrhundert und später liegt ${ }^{12}$. Für die Jahrhunderte davor ist eine monographische Aufarbeitung einzelner Legaten mangels hinreichender Quellenbasis kaum möglich ${ }^{13}$. Immerhin liegen für Legaten des 11. und 12. Jahrhunderts einige Aufsätze $\operatorname{vor}^{14}$ sowie vor allem die Veröffentli-

tits papes en voyage: Les legats en France et en Angleterre au XIII siècle, in: Se deplacer du Moyen Âge a nos jours, hg. v. Stéphane Curveiller, Calais 2008, S. 58-70. Für die Iberische Halbinsel: Ingo FleISCH: Rom und die Iberische Halbinsel: das Personal der päpstlichen Legationen und Gesandtschaften im 12. Jahrhundert, in: JOHRENDT/MülLER (wie Anm. 1) S. 135-189. Für Ostmitteleuropa: Przemysław NowaK: Das Papsttum und Ostmitteleuropa (Böhmen-Mähren, Polen, Ungarn) vom 10. bis zum beginnenden 13. Jahrhundert. Mit einer Neuedition von JL 9067, im vorliegenden Band S. 331-369. P. Nowak plant außerdem eine Monographie zu päpstlichen Legaten in Polen im 12. und 13. Jahrhundert

12 Beispielhaft sollen aus dem breiten Strom der Einzeluntersuchungen über (Kardinal-) Legaten des 13., 14. und 15. Jahrhunderts nur einige neuere Untersuchungen genannt werden: Werner MALECZEK: Petrus Capuanus. Kardinal, Legat am Vierten Kreuzzug, Theologe († 1214), Wien 1988 (Publikationen des Österreichischen Historischen Instituts in Rom 1/8) (italienische leicht erweiterte Übersetzung: Amalfi 1997); Falko NeININGER: Konrad von Urach († 1227). Zähringer, Zisterzienser, Kardinallegat, Paderborn u.a. 1994 (Quellen und Forschungen aus dem Gebiet der Geschichte N.F. 17); Claudia MÄrTL: Kardinal Jean Jouffroy († 1473). Leben und Werk, Sigmaringen 1996 (Beiträge zur Geschichte und Quellenkunde des Mittelalters 18); Nicolas VINCENT: The Letters and Charters of Cardinal Guala Bicchieri, Papal Legate in England, 1216-1218, Woodbridge 1996 (Canterbury and York Society 83).

13 Aus einer bisher unpublizierten Dissertation über Bischof Hugo von Die hat der Autor die Ergebnisse in mehreren Aufsätzen publiziert: Kriston R. RENNIE: The Application of Reform in Eleventh-Century France. The Conciliar Activity of Hugh, Bishop of Die (1073-1085), (Diss. Masch.) King's College London, 2005; DERS.: Reform in the Localities: The Council of Valence (May 1079), in: AHC 37 (2005) S. 43-55; DERS.: Collaboration and Council Criteria in the Age of Reform. Legatine Councils under Gregory VII, in: AHC 38 (2006) S. 95-118; DERS.: „Uproot and Destroy, Build and Plant". Legatine Authority under Pope Gregory VII (1073-85), in: Journal of Medieval History 33 (2007) S. 166-180; DERS.: Hugh of Die and the Legatine Office unter Gregory VII. On the Effects of a waning Administration, in: RHE 103 (2008) S. 2749.

14 Vgl. exemplarisch: Dietrich Lohrmann: Petrus von S. Grisogono und St. Viktor in Paris. Zur Vorgeschichte eines Legaten Alexanders III. in Frankreich, in: Deus qui mutat tempora. Menschen und Institutionen im Wandel des Mittelalters. Festschrift für Alfons Becker zu seinem fünfundsechzigsten Geburtstag, hg. v. Ernst-Dieter HeHL/Hubertus SEIBERT/Franz STAAB, Sigmaringen 1987, S. 259-267; Rudolf HiESTAND: Kardinalbischof Matthäus von Albano, das Konzil von Troyes [1129] und die Entstehung des Templerordens, in: ZKG 99 (1988) S. 295-325; Michael HORN: Der Kardinalbischof Imar von Tusculum als Legat in England 1144/45, in: HJb 110 (1990) S. 492-505; Ilicia SPREY: Henry of Winchester and the Expansion of Legatine Political Authority in England, in: RHE 91 (1996) S. 785-804; Gergely KIsS: Teuzo sancte 
chungen über das Kardinalskollegium und die Kurie, die stark prosopographisch ausgerichtet sind ${ }^{15}$.

Eigens hervorgehoben zu werden verdient die Arbeit von Stefan Weiß über die Urkunden der päpstlichen Legaten für den Zeitraum von 1049 bis 1198, da sie den in allen einschlägigen Darstellungen zur Diplomatik völlig vernachlässigten Urkundentypus erstmals ausführlich behandelt ${ }^{16}$. Die Ergeb-

Romane Ecclesie legatus... Teuzo cardinalis (Adalekok az I. Laszlo-kori papai magyar kapcsolatok toerttenetehez), in: Magyaroknak eleiroel. Uennepi tanulmanyok a hatvan esztendoes Makk Ferenc tiszteletere [Festschrift für Ferenc Makk zum 60. Geburtstag], hg. v. Ferenc Piti, Szeged 2000, S. 265-277; Uta-Renate Blumenthal: Hugh of Die and Lyons, Primate and Papal Legate, in: Scripturus Vitam. Lateinische Biographie von der Antike bis in die Gegenwart. Festgabe für Walter Berschin zum 65. Geburtstag, hg. v. Dorothea Walz, Heidelberg 2002, S. 487-495; Anders BERGQUIST: The Papal Legate: Nicholas Breakspear's Scandinavian Mission, in: Adrian IV, the English Pope (1154-1159). Studies and Texts, hg. v. Brenda Bolton/Anne J. DugGan, Aldershot 2003 (Church, Faith and Culture in the Medieval West), S. 41-48; Ludwig FALKENSTEIN: Wilhelm vom Champagne, Elekt von Chartres (1164-1168), Erzbischof von Sens (1168/69-1176), Erzbischof von Reims (1176-1202), Legat des apostolischen Stuhles, im Spiegel päpstlicher Schreiben und Privilegien, in: ZRGKanAbt 89 (2003) S. 107-284; DERS.: Guillaume aux Blanches Mains: archevêque de Reims et légat du Siège Apostolique (1176-1202), in: RHE 91 (2005) S. 5-25; Damian J. SMITH: The Iberian Legations of Cardinal Hyacinth Bobone, in: Pope Celestine III (1191-1198). Diplomat and Pastor, hg. v. John DORAN/Damian J. SMITH, Aldershot 2008 (Church, Faith and Culture in the Medieval West), S. 81-112.

15 Vgl. MaleczeK: Papst (wie Anm. 5) mit Erwähnung der gesamten älteren Literatur zum Kardinalat. Zu Kurie und Kanzlei vgl. die neueren Darstellungen von Georg MAY: Ego N.N. Catholicae Ecclesiae Episcopus. Entstehung, Entwicklung und Bedeutung einer Unterschriftsformel im Hinblick auf den Universalepiskopat des Papstes, Berlin 1995 (Kanonistische Studien und Texte 43); Frank M. BisCHOFF: Urkundenformate im Mittelalter. Größe, Format und Proportionen von Papsturkunden in Zeiten expandierender Schriftlichkeit (11.-13. Jahrhundert), Marburg a. d. L. 1996 (elementa diplomatica 5); Rudolf HIESTAND: Die Leistungsfähigkeit der päpstlichen Kanzlei im 12. Jahrhundert mit einem Blick auf den lateinischen Osten, in: Papsturkunde und europäisches Urkundenwesen. Studien zu ihrer formalen und rechtlichen Kohärenz vom 11. bis 15. Jahrhundert, hg. v. Peter HeRdE/Hermann JAKOBS, Köln u.a. 1999 (ADipl Beih. 7), S. 1-26; Stefan HirschmanN: Die päpstliche Kanzlei und ihre Urkundenproduktion (1141-1159), Frankfurt a. M. 2001 (Europäische Hochschulschriften 3/913); Przemysław NOWAK: Die Urkundenproduktion der päpstlichen Kanzlei 1181-1187, in: ADipl 49 (2003) S. 91-222; Bernard BARBICHE: Diplomatie, diplomatique et théologie: les préambules des lettres de légation (XIII $-\mathrm{XVII}^{\mathrm{e}}$ siècle), in: Inquirens subtilia diversa. Dietrich Lohrmann zum 65. Geburtstag, hg. v. Horst KranZ/Ludwig Falkenstein, Aachen 2002, S. 123-132. Geschichte des Kardinalats im Mittelalter, hg. v. Jürgen Dendorfer/Ralf Lützelschwab, Stuttgart 2011 (Päpste und Papsttum 39).

16 Weiss: Urkunden (wie Anm. 5); DERS.: Die Legatenurkunde des 11. und 12. Jahrhunderts zwischen Papst- und Herrscherurkunde, in: Papsturkunde und europäisches Urkundenwesen. Studien zu ihrer formalen und rechtlichen Kohärenz vom 11. bis 15 . Jahrhundert, hg. v. Peter HeRdE/Hermann JAKOBS, Köln u.a. 1999 (ADipl Beih. 7), 
nisse lassen sich in vielerlei Hinsicht für eine historische Analyse nutzbar machen $^{17}$, sie betreffen mit etwa 120 berücksichtigten Kardinälen (von denen bisher 592 Urkunden bekannt sind) höchstens ein Drittel aller zwischen 1049 und 1198 mit der Legatenwürde ausgestatteten Personen.

\section{Perspektiven der Erforschung des Legatenwesens}

Neuere Forschungsansätze zur Kommunikation, zur Medialität oder zum Rituell-Zeremoniellen, die sich für das Gesandtschaftswesen generell aufdrängen, sind für die Erforschung des Botenwesens in jüngerer Zeit facettenreich aufgenommen worden ${ }^{18}$, für das Legationswesen aber bisher nur sporadisch ${ }^{19}$. Für das 12. Jahrhundert muss diese Zurückhaltung mit der geringen Aussagekraft

S. 27-38; DERS.: Legatenurkunde und Papsturkunde, in: Hundert Jahre Papsturkundenforschung, Bilanz - Methoden - Perspektiven. Akten eines Kolloquiums zum hundertjährigen Bestehen der Regesta Pontificum Romanorum vom 9.-11. Oktober 1996 in Göttingen, hg. v. Rudolf HIESTAND, Göttingen 2003, (AAG, 3. Folge 261), S. 335-350 mit sieben Nachträgen. In der zweiten Auflage des Handbuchs von Thomas FrENZ: Papsturkunden des Mittelalters und der Neuzeit, akt. Aufl., Stuttgart ${ }^{2} 2000$ (Historische Grundwissenschaften in Einzeldarstellungen 2), S. 115, ist den Legatenurkunden immerhin eine halbe Seite gewidmet.

17 Vgl. Claudia ZEY: Gleiches Recht für alle? Konfliktlösung und Rechtsprechung durch päpstliche Legaten im 11. und 12. Jahrhundert, in: Rechtsverständnis und Konfliktaustragung im Mittelalter, hg. v. Stefan ESDERS, Köln u.a. 2007, S. 93-119.

18 Vgl. Jürg Zulliger: „Ohne Kommunikation würde Chaos herrschen“. Zur Beobachtung von Informationsaustausch, Briefverkehr und Boten bei Bernhard von Clairvaux, in: AK 78 (1996) S. 251-276; Bernhard Siegert: Vögel, Engel und Gesandte. Alteuropas Übertragungsmedien, in: Gespräche - Boten - Briefe. Körpergedächtnis und Schriftgedächtnis im Mittelalter, hg. v. Horst WenZeL, Berlin 1997 (Philologische Studien und Quellen 143), S. 45-62; Horst WenZeL: Boten und Briefe. Zum Verhältnis körperlicher und nichtkörperlicher Nachrichtenträger, in: DERS. (wie oben) S. 86-105; Stephan FrEUND: Boten und Briefe. Formen und Wege bayerischitalienischer Kommunikation in Früh- und Hochmittelalter, in: Bayern und Italien. Politik, Kultur, Kommunikation (8.-15. Jahrhundert). Festschrift für Kurt Reindel zum 75. Geburtstag, hg. v. Heinz DopsCH/Stephan Freund/Alois ScHMID, München 2001 (ZBLG Beih. 18, Reihe B), S. 55-103; Volker SCIOR: Veritas und certitudo oder: Warten auf Wissen. Boten in frühmittelalterlichen Informationsprozessen, in: Das Mittelalter 11 (2006) Heft 1: Engel und Boten, S. 110-131.

19 In den jüngsten Sammelbänden zu Inszenierung und Ritual spielen Themen aus dem Bereich des geistlichen und weltlichen Gesandtschaftswesens keine Rolle: Die Welt der Rituale. Von der Antike bis heute, hg. v. Claus AmBos u. a, Darmstadt 2005; Inszenierung und Ritual in Mittelalter und Renaissance, hg. v. Andrea vON HÜlSENESCH, Düsseldorf 2005 (Studia humaniora 40). 
der Quellen zu diesen Fragen begründet werden ${ }^{20}$, für das 13. Jahrhundert mit den fehlenden Vorarbeiten, die zugleich eine sinnvolle Eingrenzung des umfangreichen Stoffs ermöglichen könnten. Die bisher vorliegenden Beiträge haben daher eher einen eklektischen Zugriff auf das einschlägige Quellenmaterial des 13. Jahrhunderts mit deutlicher Bevorzugung der normativen Quellen ${ }^{21}$. Ma Tapio Salminen orientierte sich in seinem bereits vor über 20 Jahren publizierten Beitrag zum Wesen der päpstlichen Repräsentation durch Legaten nahezu ausschließlich am Dekretalenrecht und dessen gelehrter Aufarbeitung und ging nur auf wenigen Seiten auf die äußeren Repräsentationszeichen (Kleidung, Insignien) und damit auf den performativen Aspekt der Legatentätigkeit ein ${ }^{22}$. Ausgewählte Empfehlungs- und Beglaubigungsschreiben der Päpste waren für Thérèse Bœspflug neben dem Dekretalenrecht die Grundlage ihrer Überlegungen zur Begrifflichkeit sowie zum Wesen und zu den Grenzen von ,Repräsentation' als von den Päpsten kontrollierter Stellvertretung durch

$20 \mathrm{Zu}$ dem einzigen bemerkenswert frühen Zeugnis zum äußeren Erscheinungsbild eines päpstlichen Legaten in Thangmars Vita Bernwardi vgl. SALMinen (wie Anm. 5) S. 351.

21 Für einen streng kanonistisch-normativen Zugang zur Thematik stehen in jüngerer Zeit die Arbeiten von: Richard A. SCHMUTZ: Medieval Papal Representatives: Legates, Nuncios and Judges Delegate, in: Post Scripta. Essays on Medieval Law and the Emergence of the European State in Honor of Gaines Post, hg. v. Joseph R. STRAYER/Donald E. QuelLeR, Rom 1972 (Studia Gratiana 15), S. 441-463; Robert Charles Figueira: The Canon Law of Medieval Papal Legation. A Thesis presented to the Faculty of the Graduate School of Cornell University in Partial Fulfillment for the Degree of Doctor of Philosophy, (Diss. Masch.) New York 1980 (Ann Arbor Microfilms, Michigan 1980); DERS.: The Classification of Medieval Papal Legates in the „Liber Extra“, in: AHP 21 (1983) S. 211-228; DERS.: Decretalists, Medieval Papal Legation, and the Roman Law of Offices and Jurisdiction, in: Res publica litterarum. Studies in the Classical Tradition 9 (1986) S. 119-135 (auch in: Studi Umanistici Piceni 6 [1986] S. 119-136); DERS.: „Legatus apostolice sedis“: the Pope's ,,alter ego“ according to Thirteenth-Century Canon Law, in: Studi medievali $3^{\text {a }}$ serie 27 (1986) S. 527-574; DERS.: The Medieval Papal Legate and His Province. Geographical Limits of Jurisdiction, in: Apollinaris 61 (1988) S. 817-860 (auch in: Plenitude of Power. The Doctrines and Exercise of Authority in the Middle Ages. Essays in Memory of Robert Louis Benson, hg. v. DEMS., Aldershot/Burlington, VT 2006 [Church, Faith and Culture in the Medieval West], S. 73-105); DERS.: Papal Reserved Powers and the Limitations on Legatine Authority, in: Popes, Teachers, and Canon Law in the Middle Ages, hg. v. James Ross SweEnEy/Stanley ChOdorow, Ithaca (New York)/London 1989, S. 191-211; DERS.: Subdelegation by Papal Legates in Thirteenth-Century Canon Law: Powers and Limitations, in: In Iure Veritas. Studies in Canon Law in Memory of Schafer Williams, hg. v. Steven B. Bowman/Blanche E. Cody, Cincinnati 1991, S. 56-79; DERS.: Papal Reserved Powers - Some Decretist Texts, in: Grundlagen des Rechts. Festschrift für Peter Landau zum 65. Geburtstag, hg. v. Richard H. HelmHOlz u. a. (Rechts- und Staatswissenschaftliche Veröffentlichungen der Görres-Gesellschaft, NF 91), Paderborn u.a. 2000, S. 477-490.

22 SAlminen (wie Anm. 5). 
die Legaten ${ }^{23}$. Pascal Montaubin hat zur Untersuchung der Reiseumstände der Legaten überdies noch zeremonielle Zeugnisse, teilweise aus dem 14. und 15. Jahrhundert, herangezogen ${ }^{24}$.

Damit sind vielversprechende Wege für die weitere Analyse des päpstlichen Legatenwesens aufgezeigt. Dass sie fast ausnahmslos ins 13. Jahrhundert und darüber hinaus weisen, ist vor allem der Quellenlage geschuldet. Auch wenn deren systematische Erschließung schon rein quantitativ eine Herausforderung darstellt, werden sich bei entschlossenem Zugriff zumal auf das edierte Material weitere Einblicke in die strukturellen und zeremoniellen Umstände öffnen. Analog etwa zu den wertvollen Erkenntnissen, die sich aus dem Register Gregors VII. zu Theorie und Praxis des Legatenwesens am Beginn der Reformära gewinnen lassen, gewährt das Register Innozenz' III. tiefe Einblicke in die Entwicklung der Institution zu Beginn der von Juristenpäpsten geprägten Ära.

Welches Pontential in der Durchdringung des urkundlichen bzw. brieflichen Quellenmaterials liegt, konnte Werner Maleczek im Rahmen zweier größerer Untersuchungen zum Frieden stiftenden Papsttum und zur Bedeutung des Papsttums für die Universitätsgründungen im 13. Jahrhundert aufzeigen $^{25}$. In beiden Bereichen waren selbstverständlich Kardinallegaten mit entsprechendem Kenntnis- und Erfahrungsschatz die ausführenden Organe.

Die Universitäts- und Bildungsgeschichte sind ein weiteres bisher noch wenig beackertes Feld für die Legatenforschung ${ }^{26}$. Während Maleczek das Beziehungsgeflecht zwischen dem Papsttum und den französischen, englischen und italienischen Universitäten beleuchtete, zeigte Ingo Fleisch in seiner Monographie die Schlüsselrolle der päpstlichen Legaten im westiberischen Raum auf ${ }^{27}$. Für diese Region könnten sich auch für das 12. Jahrhundert mit dem weiteren Voranschreiten der Arbeiten an der Iberia Pontificia noch Verfeinerungen des derzeitigen Forschungsstandes ergeben ${ }^{28}$.

23 Thérèse B(ESPFLUG: La représentation du pape au moyen âge. Les légats pontificaux au XIII ${ }^{\mathrm{e}}$ siècle, in: MEFR 114 (2002) S. 59-71.

24 Montaubin (wie Anm. 11).

25 Werner Maleczek: Das Frieden stiftende Papsttum im 12. und 13. Jahrhundert, in: Träger und Instrumentarien des Friedens im hohen und späten Mittelalter, hg. v. Johannes FRIED, Sigmaringen 1996 (VuF 43), S. 249-332; Werner MALECZEK: Das Papsttum und die Anfänge der Universität im Mittelalter, in: RHMitt 27 (1985) S. 85143.

$26 \mathrm{Zu}$ den Beziehungen zwischen dem Papsttum bzw. dem Kardinalat und den hohen Schulen von Paris vgl. weiterhin vor allem die Arbeiten von Peter CLASSEN: Studium und Gesellschaft im Mittelalter, hg. v. Johannes FRIED, Stuttgart 1983 (MGH Schriften 29).

27 Ingo FleisCH: Sacerdotium - Regnum - Studium. Der westiberische Raum und die europäische Universitätskultur im Hochmittelalter. Prosopographische und rechtsgeschichtliche Studien, Berlin 2006 (Geschichte und Kultur der iberischen Welt 4).

28 Die in Lissabon veranstaltete Tagung „El papado limitado. Legados - jueces - limites“ (wie Anm. 2) diente der Standortbestimmung des Unternehmens unter dem speziellen 
Ein weiterer ebenfalls von Werner Maleczek jüngst aufbereiteter Zweig der Legatenforschung betrifft die ostentative Seite der repräsentativen Vollmachten: die Siegel der Kardinäle bzw. der Kardinallegaten ${ }^{29}$. Diese zunächst bis zum Beginn des 13. Jahrhunderts publizierte Studie wird Maleczek selbst fortführen, um aus einer reicheren Überlieferungslage heraus die ikonographischen Entwicklungen zu verfolgen und den Siegelgebrauch im historischen Kontext zu analysieren. Vorarbeiten existierten praktisch nicht, denn ebenso wie die Legatenurkunden wurden auch die Legatensiegel in der hilfswissenschaftlichen Forschung stark vernachlässigt.

Der Versuch, eine bestimmte Richtung zu prognostizieren, in welche die Erforschung des päpstlichen Legatenwesens in den kommenden Jahren gehen wird oder gehen sollte, würde schon an der Vielfalt der derzeit verfolgten Forschungsansätze scheitern. Bewährte geographische und prosopographische Ansätze werden vertieft, neuere medienwissenschaftliche Ansätze werden aufgegriffen. Beachtlich und erfreulich zugleich ist dabei die Internationalität der Forschung und der rege Austausch unter den Forscherinnen und Forschern. Hélène Millet und Pascal Montaubin gelang es für ihre Legatentagung in Paris im Februar 2009, 27 Forscherinnen und Forscher aus zehn Ländern zusammenzuführen und zahlreiche Aspekte des päpstlichen Legatenwesens erörtern zu lassen ${ }^{30}$.

Internationalen Werkstattcharakter haben ebenfalls die vor allem von Maria Pia Alberzoni initierten Arbeitstreffen in Mailand zur Erforschung des Legatenwesens und der delegierten Gerichtsbarkeit. Nach einer ersten Sichtung des Themenspektrums im Jahr $2009^{31}$ ging es bei der Zusammenkunft im Frühjahr 2011 um die Bedeutung der päpstlichen Legaten und der delegierten Richter für die Kreuzfahrerherrschaften. Damit wird ein weiterer Aspekt berührt, der in der internationalen Forschungsperspektive bisher nur eine marginale Rolle gespielt hat, nämlich die Frage nach der Bedeutung der päpstlichen Legaten für den inner- und außereuropäischen Kultur- und Wissenstransfer.

Blickwinkel ,Legaten und delegierten Richter'. Zum Planungs-und Bearbeitungsstand vgl. <http://www.papsturkunden.gwdg.de/Pius-Stiftung/Publikationen/publikationen. html\#Iberia $>$ und < http://www.papsturkunden.gwdg.de/Akademieprojekt/__ Iberia /__iberia__. html>.

29 Werner MaleczeK: Die Siegel der Kardinäle. Von den Anfängen bis zum Beginn des 13. Jhs., in: MIÖG 112 (2004) S. 177-203.

30 „Les légats pontificaux. Paix et unité de l'Eglise, de la restructuration grégorienne à l'aube du Concile de Trente (mi XI ${ }^{\mathrm{e}}-\mathrm{mi} \mathrm{XVI}^{\mathrm{e}}$ siècle)“ (wie Anm. 2).

31 „Legati e delegati: le carriere e gli ambiti di azione (secoli XII-XIII) / Päpstliche Legaten und Delegaten: Forschungsstand und Forschungsfragen (12.-13. Jahrhundert)" (wie Anm. 2) 